Supporting Information for

\title{
Statistical Time-resolved Spectroscopic Study on Upconversion Luminescence
}

Youngho Cho, ${ }^{\dagger, t^{*}}$ Soo Yeong Lim, ${ }^{\dagger *}$ Song Yeul Lee, ${ }^{\S}$ Jae Hun Kim, ${ }^{\ddagger}$ Yong Il Park, ${ }^{\S}$ Chan Ryang Park ${ }^{\dagger}$ and Hyung Min Kim*,

†Department of chemistry, Kookmin University, Seoul, 02707, Republic of Korea

‡Sensor Sy stem Research Center, Korea Institute of Science and Technology (KIST), Seoul, 02792, Republic of Korea

§School of Chemical Engineering, Chonnam National University, Gwangju, 61186, Republic of Korea

*These authors contributed equally to this work.

*Correspondence :

Prof. Hyung Min Kim

Department of Chemistry

Kookmin University

77 Jeongneung-ro, Seongbuk-gu, Seoul, 02707, Republic of Korea

E-mail : hyungkim@kookmin.ac.kr 
Figure S1. (a) Experimental pulse-modulated spectroscopy setup with triple photodetectors, (PD: a photodiode (DET36A/M, Thorlabs), PMT: a photomultiplier tube (R928, HAMAMATSU), and SP: a spectrometer (SM245, Korea Spectral Products) and 980nm laser (QFBGLD-980-250, QPHOTONICS), pulse generator (DG535, Stanford Research Systems,INC), oscilloscope (LT372, LeCroy), monochromator (250is, Chromex). (b) photograph of 980nm ON/OFF.

(a)

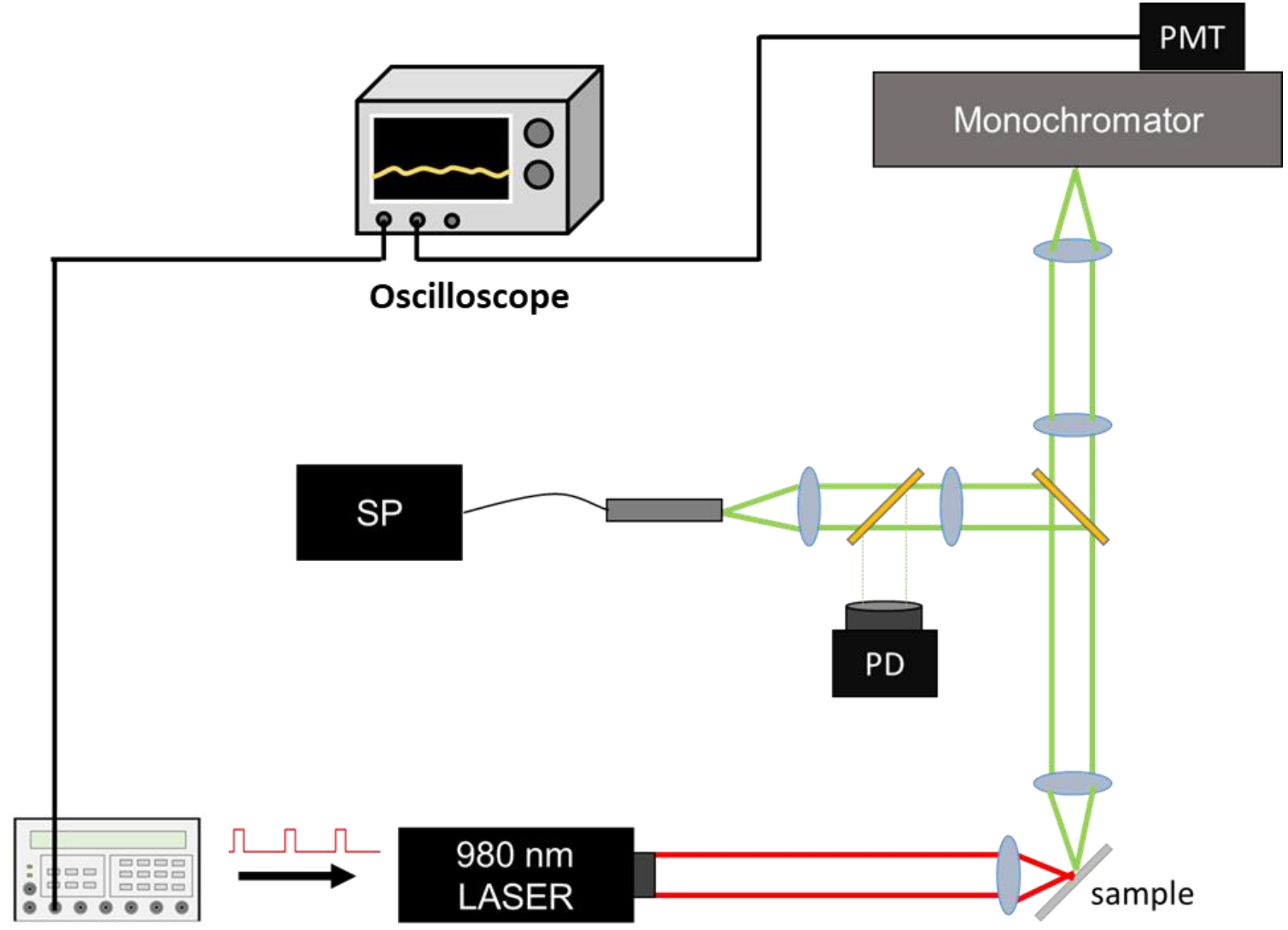

\section{Pulse Generator}

(b)
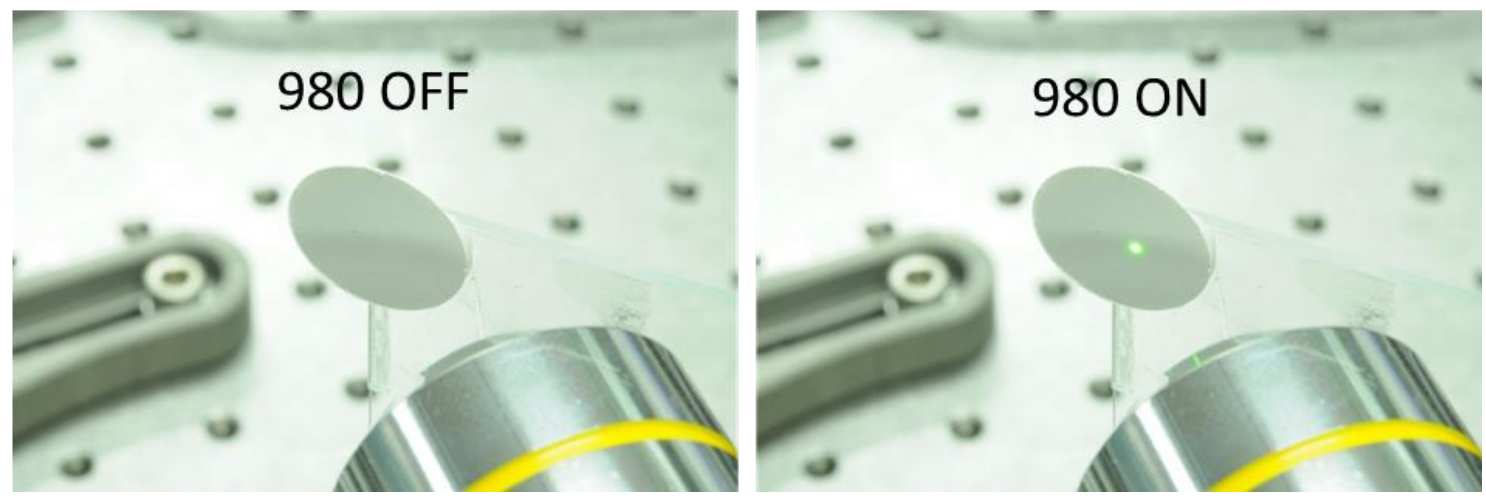
Figure S2. Excitation pulse waveforms for pulse-width control experiments. (a), (c), (e) Time-domain and (b), (d), (f) frequency-domain pulse-modulated spectra of the upconversion phosphor with three different pulse heights.

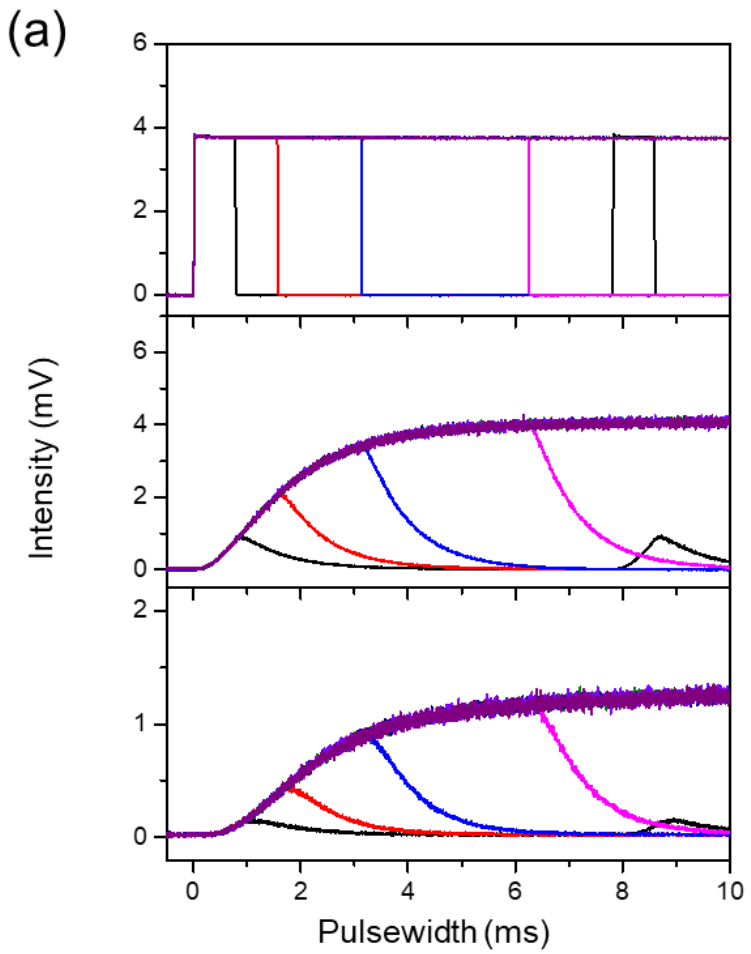

(b)

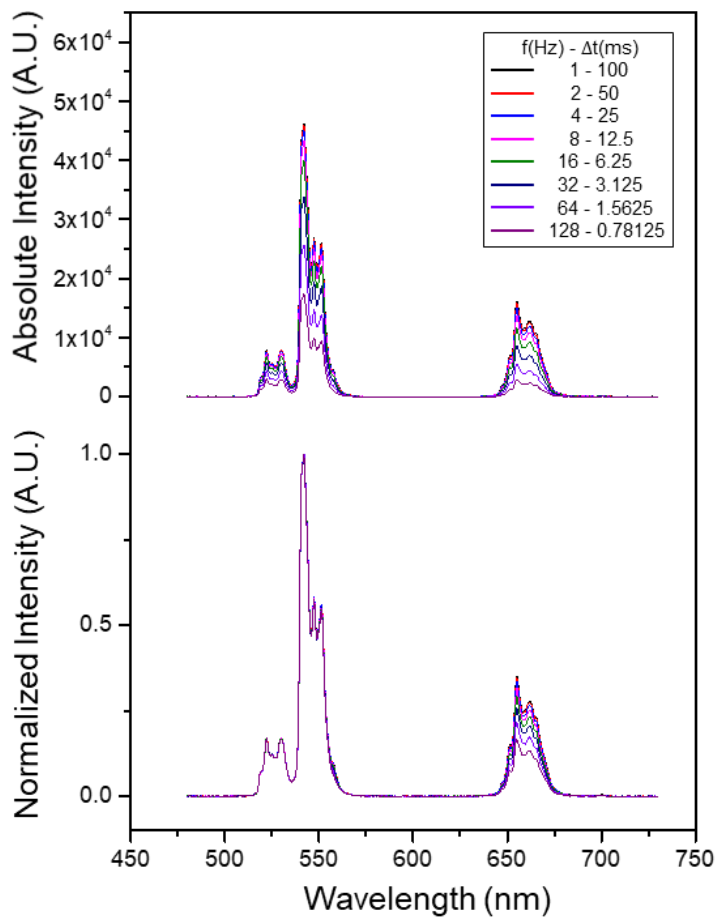

(c)

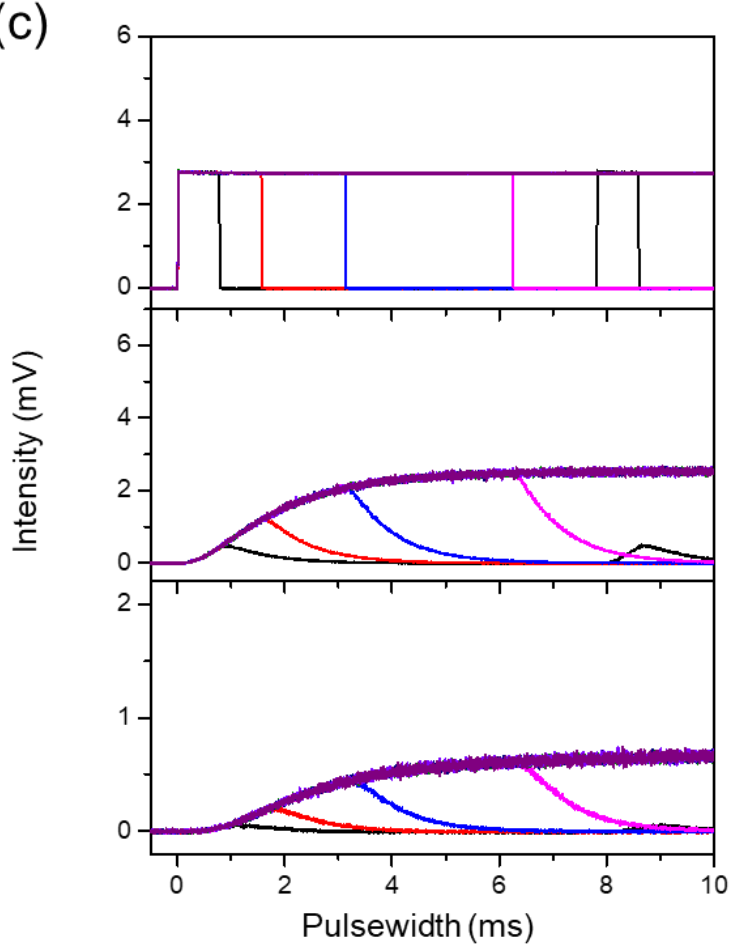

(d)

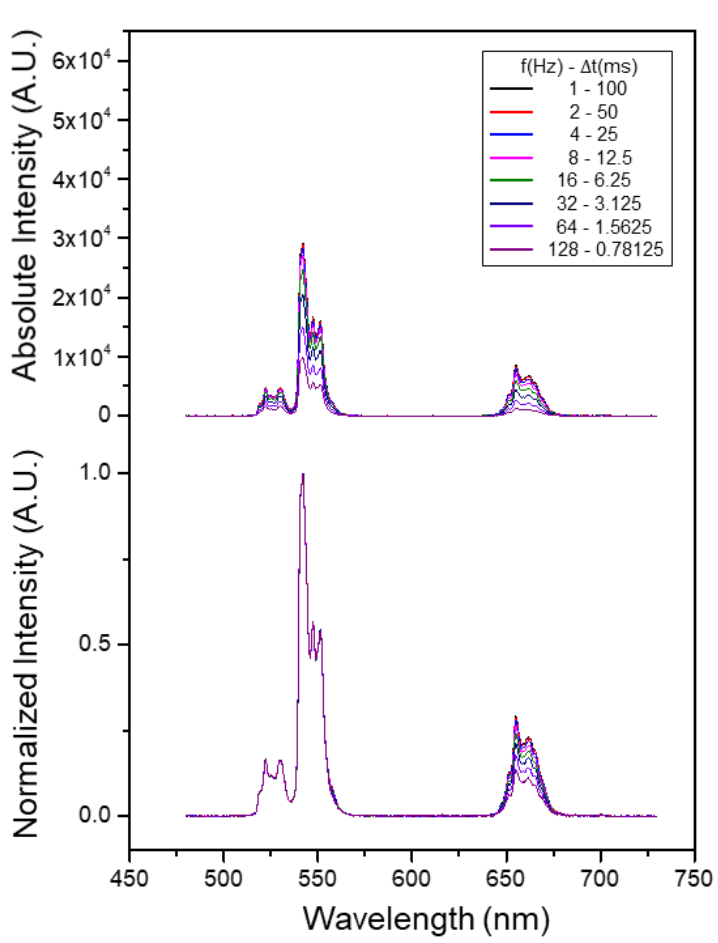



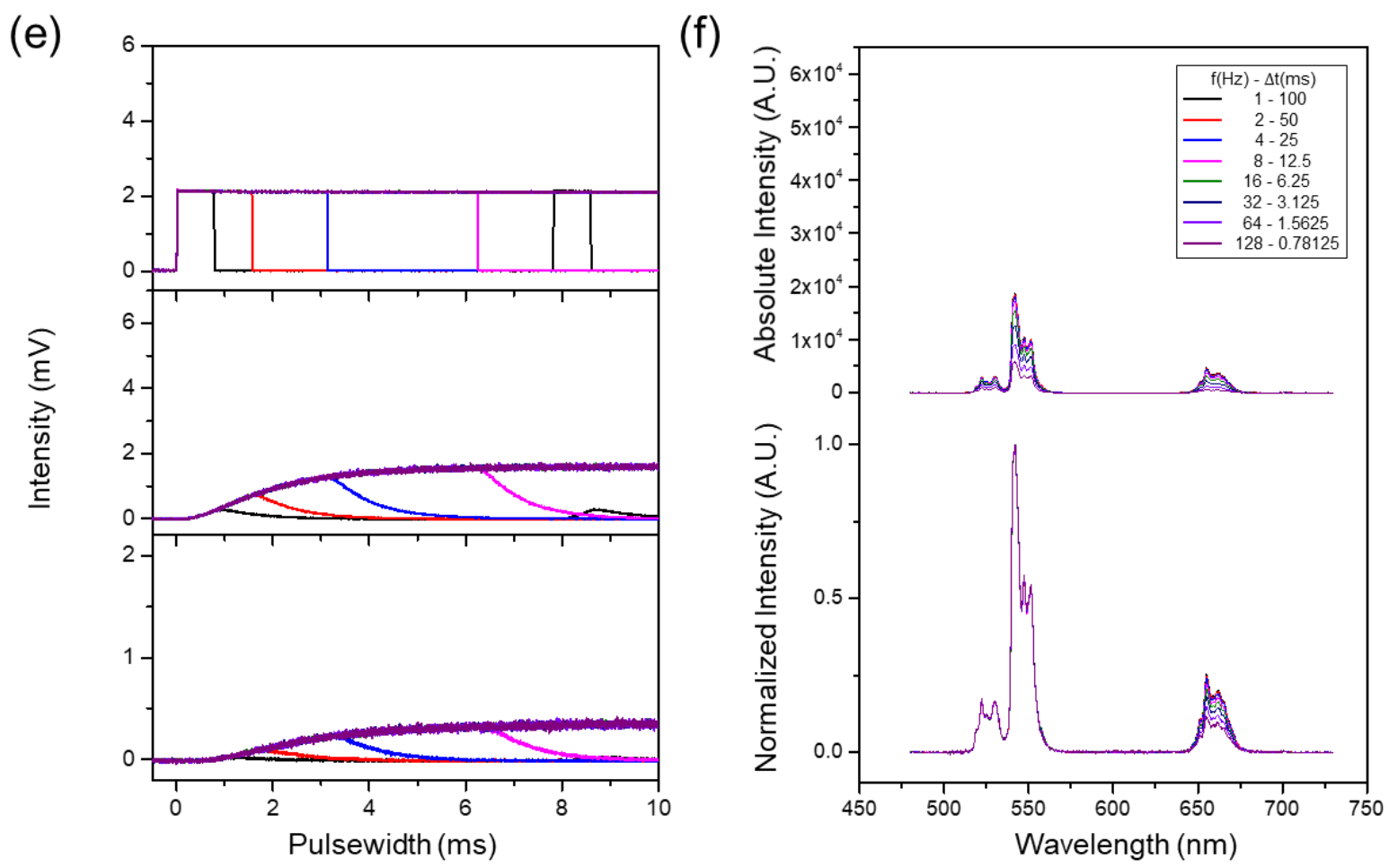
Figure S3. Excitation pulse waveforms for pulse-interval control experiments. (a), (c), (e) Time-domain and (b), (d), (f) frequency-domain pulse-modulated spectra of the upconversion phosphor with three different pulse heights.

(a)

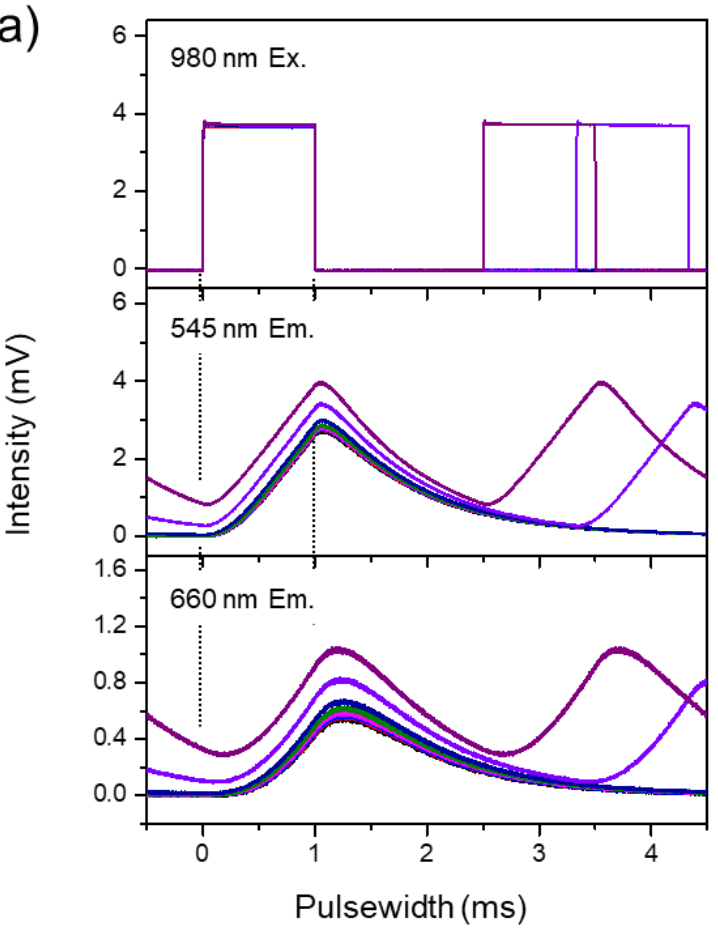

(c)

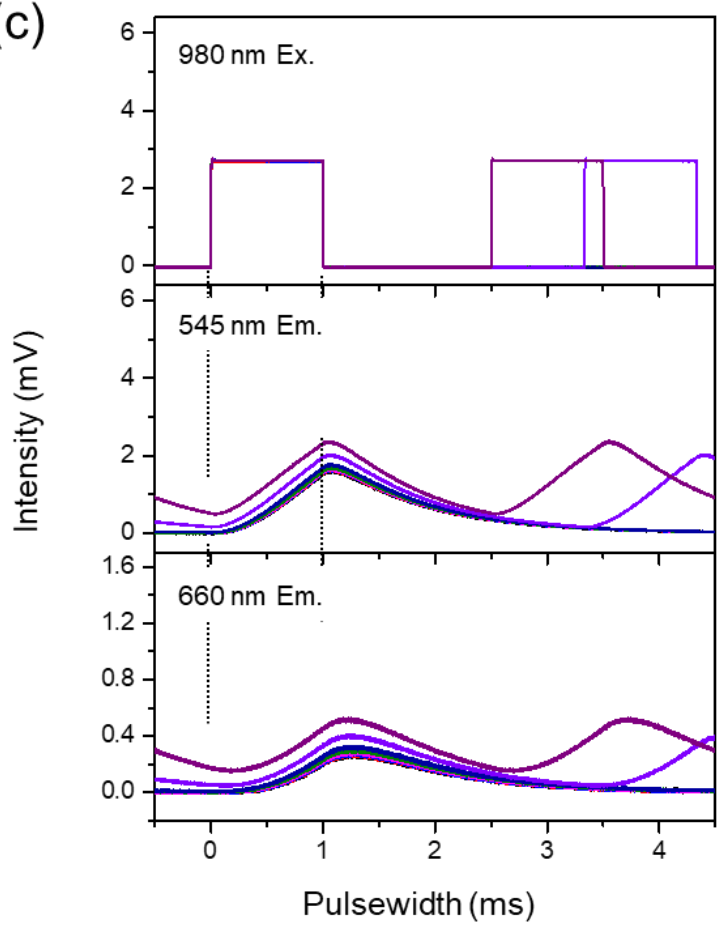

(b)

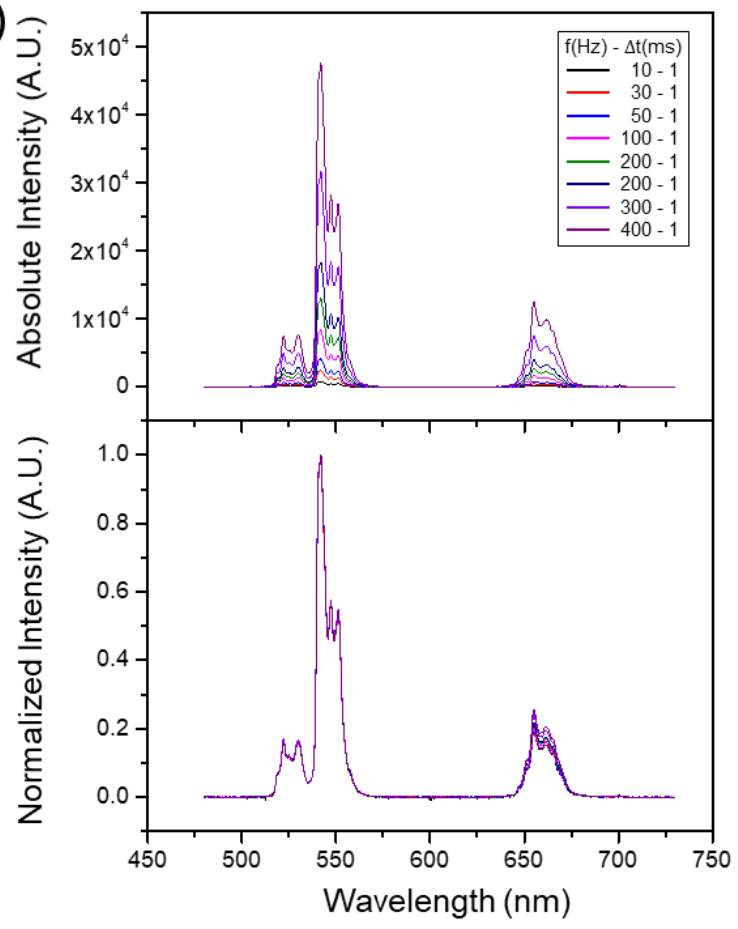

(d)

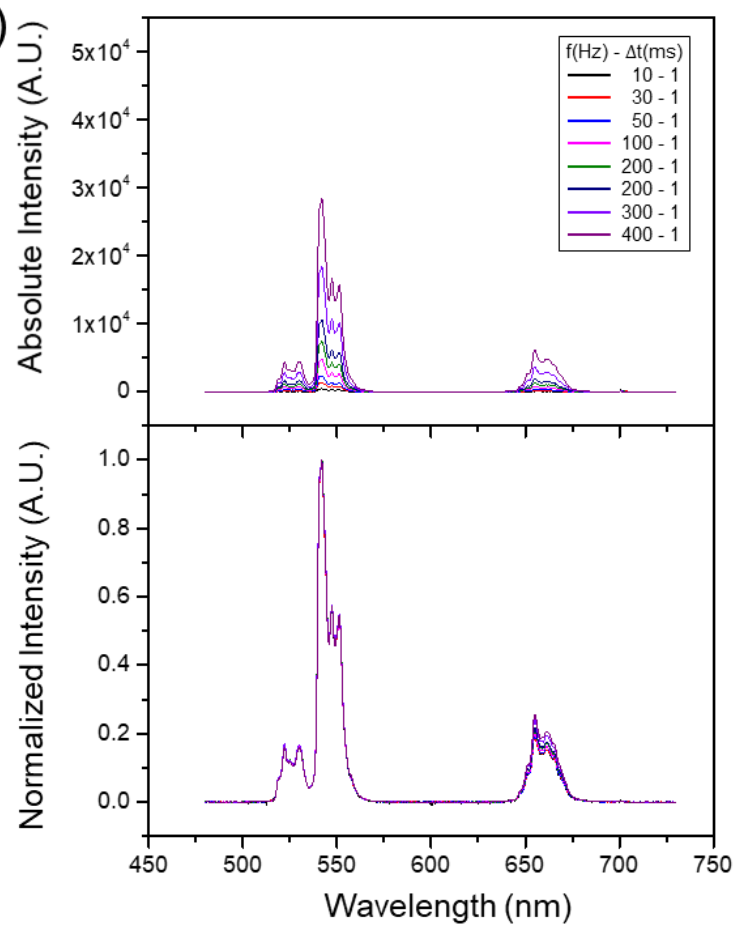


(e)

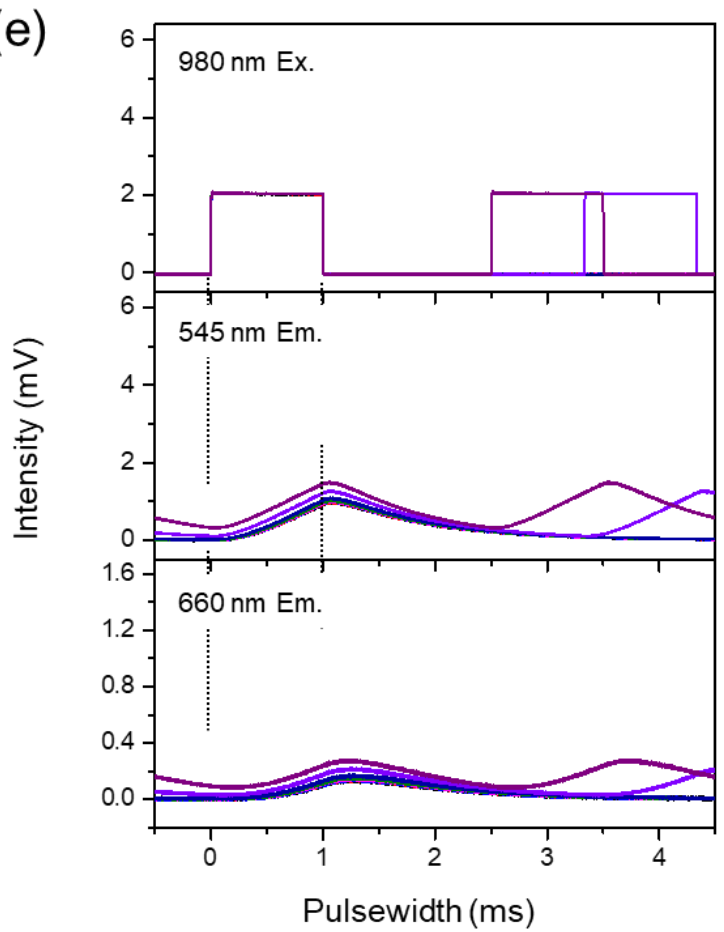

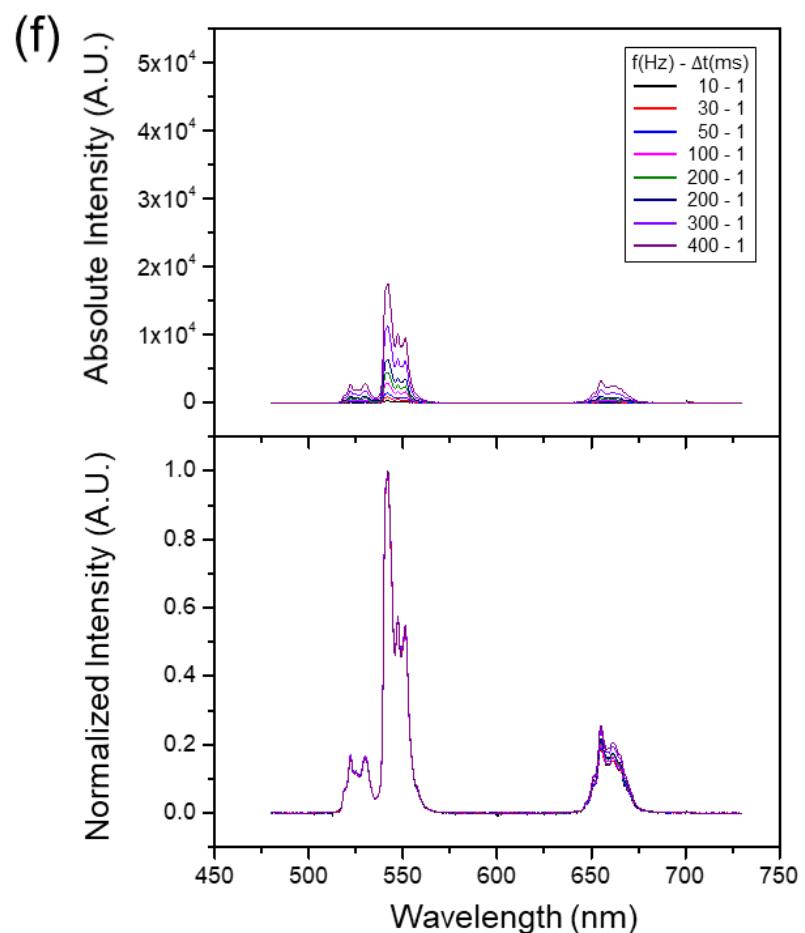


Figure S4. (a) Experimental pulse-modulated spectroscopy setup of 980+1532nm, (PD: a photodiode (DET36A/M, Thorlabs), PMT: a photomultiplier tube (R928, HAMAMATSU), and SP: a spectrometer (SM245, Korea Spectral Products) and 980nm laser (QFBGLD-980-250, QPHOTONICS), 1532nm laser (MDL-N-1532-1W, CNI Laser, pulse generator (DG535, Stanford Research Systems,INC), oscilloscope (LT372, LeCroy), monochromator (250is, Chromex). (b) Luminescence spectra of $\mathrm{NaYF}_{4}: \mathrm{Yb}^{3+}, \mathrm{Er}^{3+}$ under $980 \mathrm{~nm}$ and $1532 \mathrm{~nm}$ irradiation, separately; $1532 \mathrm{OFF}$ (black line), 1532nm excitation (red line), spectra of optical sum (blue line), subtract 1532 ON to 1532 OFF (magenta line). (c) photograph of 1532 ON.

(a)
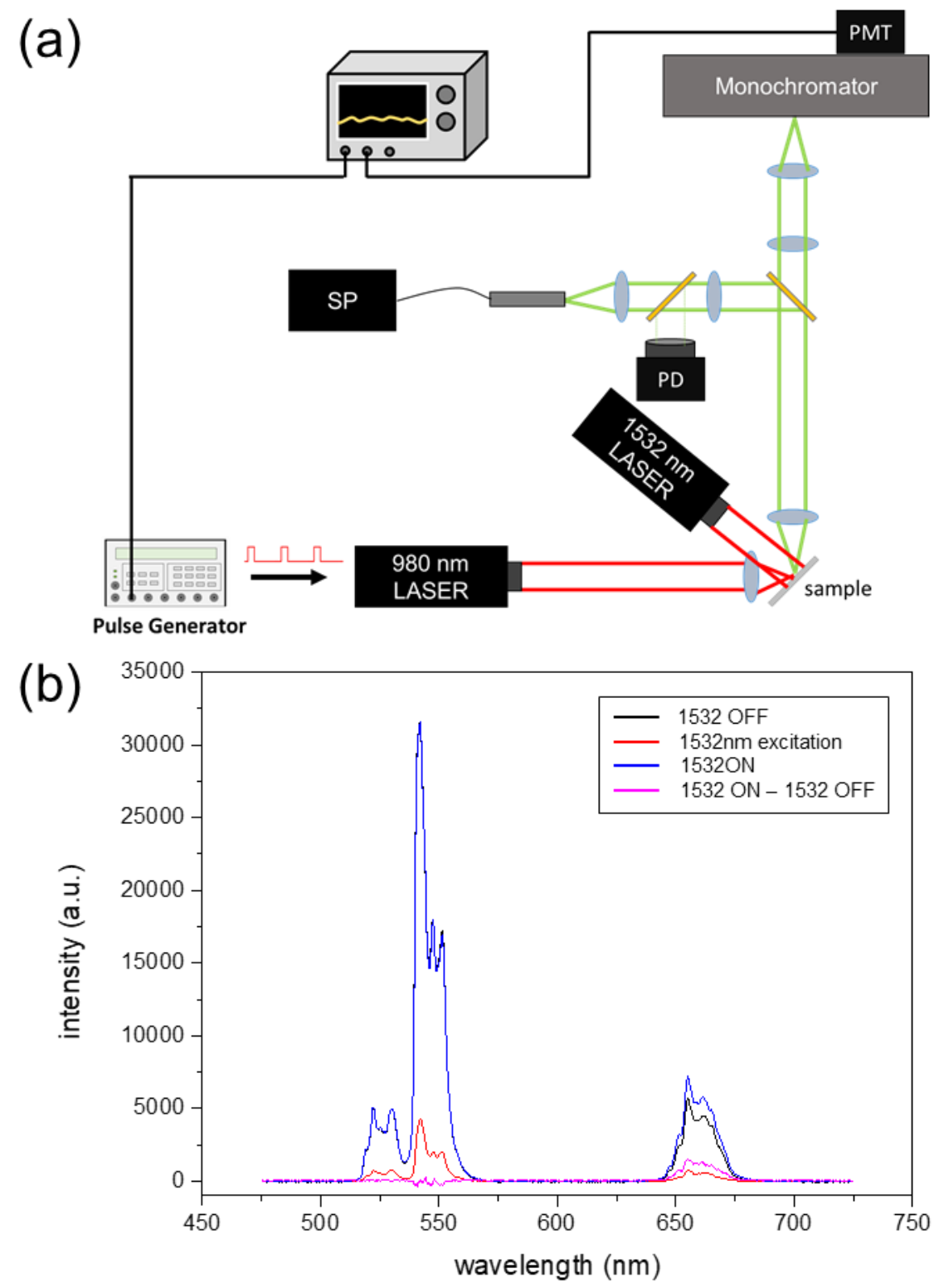
(c)

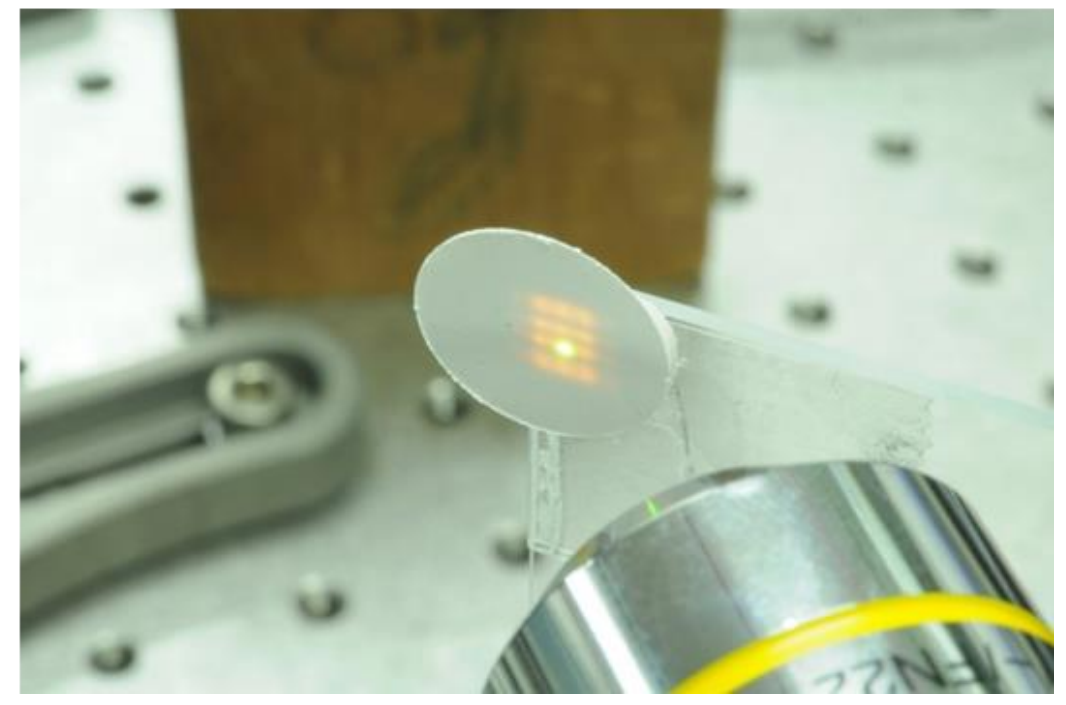


Figure S5. Synthesis method of upconversion nanoparticle.

Reagents

Erbium acetate hydrate (99.9\%, Aldrich), ytterbium acetate hydrate (99.9\%, Aldrich), yttrium acetate hydrate (99.9\%, Aldrich), sodium hydroxide (97\%, Aldrich), ammonium fluoride (99.9\%, Aldrich), 1 octadecene (ODE) (>90\%, Aldrich), oleic acid (OA) (>90\%, Aldrich)

Synthesis

Upconversion nanoparticles (UCNPs) were prepared according to reported procedure with minor modification (Adv. Mater. 2012, 24, 5755). Yttrium acetate hydrate $(0.78 \mathrm{mmol})$, ytterbium acetate hydrate $(0.20 \mathrm{mmol})$, and erbium acetate hydrate $(0.02 \mathrm{mmol})$ were added into a three-necked flask containing OA $(10 \mathrm{~mL})$ and $\mathrm{ODE}(15 \mathrm{~mL})$. The solution was heated to $150{ }^{\circ} \mathrm{C}$ and kept it for $40 \mathrm{~min}$, and then cooled to room temperature. Sodium hydroxide $(2.5 \mathrm{mmol})$ and ammonium fluoride $(4.0 \mathrm{mmol})$ was dissolved in methanol, respectively. Each methanol solution was added to the reactor and stirred for 30 min at $50{ }^{\circ} \mathrm{C}$. The mixture was heated to $110{ }^{\circ} \mathrm{C}$ under vacuum with stirring for $15 \mathrm{~min}$ to remove methanol. The flask was switched to Ar atmosphere and the temperature was raised to $310{ }^{\circ} \mathrm{C}$ at a rate of $10{ }^{\circ} \mathrm{C} / \mathrm{min}$ and maintained for an hour. Finally, the reactor was cooled to room temperature, and the nanoparticles were precipitated by addition of ethanol, and recovered by centrifugation. The product was dispersed in cyclohexane and stored in refrigerator. 
Figure S6. Experimental pulse-modulated spectroscopy setup of 980+1532nm of upconversion nanoparticle. SP: a spectrometer (SM245, Korea Spectral Products), 980nm laser (QFBGLD-980-250, QPHOTONICS), 1532nm laser (MDL-N-1532-1W, CNI Laser, pulse generator (DG535, Stanford Research Systems,INC).
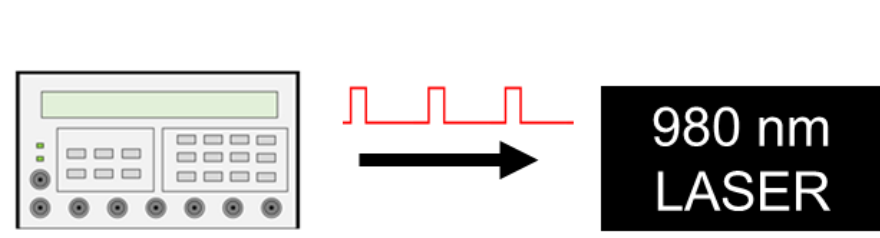

\section{Pulse Generator}

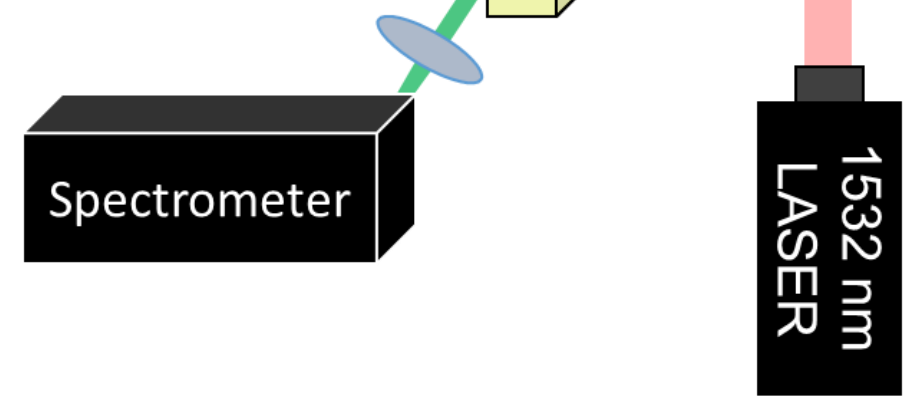

